\title{
Block intersection polynomials
}

\author{
Peter J. Cameron and Leonard H. Soicher \\ School of Mathematical Sciences \\ Queen Mary, University of London \\ Mile End Road, London E1 4NS, UK
}

P.J.Cameron@qmul.ac.uk, L.H.Soicher@qmul.ac.uk

September 8, 2006

\begin{abstract}
We introduce the block intersection polynomial, which is constructed using certain information about a block design with respect to a subset $S$ of its point-set, and then provides further information about the number of blocks intersecting $S$ in exactly $i$ points, for $i=0, \ldots,|S|$. We also discuss some applications of block intersection polynomials, including bounding the multiplicity of a block in a $t$ - $(v, k, \lambda)$ design and in a resolvable $t-(v, k, \lambda)$ design.
\end{abstract}

\section{Introduction}

This paper introduces the block intersection polynomial, which is constructed using certain information about a block design with respect to a subset $S$ of its point-set, and then provides further information about the number of blocks intersecting $S$ in exactly $i$ points, for $i=0, \ldots,|S|$. We also discuss some applications of block intersection polynomials, including bounding the multiplicity of a block in a $t$ - $(v, k, \lambda)$ design and in a resolvable $t$ - $(v, k, \lambda)$ design. Certain special cases of the results presented here, and related results, appear in [5] and in [3]. 


\section{Block designs}

A block design is an ordered pair $(V, \mathcal{B})$, such that $V$ is a finite non-empty set, whose elements are called points, and $\mathcal{B}$ is a finite non-empty multiset of nonempty subsets of $V$ called blocks. (Statisticians would call our block designs binary block designs (see [1]).) A block design is simple if each block has multiplicity 1 (that is, there are no repeated blocks). A parallel class of a block design is a set of blocks forming a partition of the point-set. A block design is resolvable if there is a partition of the blocks, each part of which is a parallel class. For $t$ a non-negative integer and $v, k, \lambda$ positive integers with $t \leq k \leq v$, a $t$ - $(v, k, \lambda)$ design (or simply a $t$-design) is a block design with exactly $v$ points, such that each block has size $k$ and each $t$-subset of the point-set is contained in exactly $\lambda$ blocks.

Now let $D=(V, \mathcal{B})$ be a block design, and $S$ a subset of $V$, with $s=|S|$. We are interested in determining information about the number $n_{i}$ of blocks of $D$ that intersect $S$ in exactly $i$ points (counting repeated blocks), for $i=0, \ldots, s$, given certain known (or proposed) properties of the pair $D, S$. Of particular interest are when $S$ is a block with a given multiplicity and when $D$ is resolvable.

For a subset $T$ of the point-set of $D$, define $\lambda_{T}$ to be the number of blocks of $D$ (counting repeats) containing $T$, and for $0 \leq j \leq s$, define

$$
\lambda_{j}=1 /\left(\begin{array}{l}
s \\
j
\end{array}\right) \sum_{T \subseteq S,|T|=j} \lambda_{T} .
$$

Then the required known properties of $D, S$ are:

- non-negative integers $m_{0}, \ldots, m_{s}$ such that $m_{i} \leq n_{i}$ (the closer $m_{i}$ is to $n_{i}$, the better);

- for some even $t \leq s$, the quantities $\lambda_{0}, \ldots, \lambda_{t}$ (the larger $t$ is, the better).

For example, if $S$ is a block, then we can take $m_{s}=1$, and more generally, if $S$ is a block of multiplicity at least $n$, then we can take $m_{s}=n$. If $S$ is a block in a parallel class and all blocks have size $s$ (dividing $|V|$ ), then we can take $m_{0}=|V| / s-1$. If nothing is known about an $m_{i}$ then we can take $m_{i}=0$. We also observe that $\lambda_{0}, \ldots, \lambda_{t}$ are easy to determine if $D$ is a $t-(v, k, \lambda)$ design and $t \leq s$; then for $j=0, \ldots, t, \lambda_{j}=\lambda\left(\begin{array}{c}v-j \\ t-j\end{array}\right) /\left(\begin{array}{c}k-j \\ t-j\end{array}\right)$.

Now, for $0 \leq j \leq s$, let us count in two ways the number $N_{j}$ of ordered pairs $(B, T)$ such that $B$ is a block of $D$ and $T$ is an $j$-subset of both $S$ and $B$. 
1. Each $j$-subset $T$ of $S$ contributes exactly $\lambda_{T}$ pairs of the form $(*, T)$ to $N_{j}$, and so

$$
N_{j}=\sum_{T \subseteq S,|T|=j} \lambda_{T}=\left(\begin{array}{l}
s \\
j
\end{array}\right) \lambda_{j} .
$$

2. Each block $B$ contributes exactly $\left(\begin{array}{c}|B \cap S| \\ j\end{array}\right)$ pairs of the form $(B, *)$ to $N_{j}$, and so

$$
N_{j}=\sum_{B}\left(\begin{array}{c}
|B \cap S| \\
j
\end{array}\right)=\sum_{i=0}^{s}\left(\begin{array}{l}
i \\
j
\end{array}\right) n_{i} .
$$

We conclude that

$$
\left(\begin{array}{l}
s \\
j
\end{array}\right) \lambda_{j}=\sum_{i=0}^{s}\left(\begin{array}{l}
i \\
j
\end{array}\right) n_{i} .
$$

\section{Polynomials}

For $k$ a non-negative integer, define the polynomial

$$
P(x, k)=x(x-1) \cdots(x-k+1) .
$$

Thus, for $n$ a non-negative integer, $\left(\begin{array}{l}n \\ k\end{array}\right)=P(n, k) / k$ !.

Lemma 3.1 Let $a, b, t$ be non-negative integers. Then

$$
P(a+b, t)=\sum_{j=0}^{t}\left(\begin{array}{l}
t \\
j
\end{array}\right) P(a, j) P(b, t-j) .
$$

Proof. Look at the coefficient of $x^{t}$ in

$$
(1+x)^{a+b}=(1+x)^{a}(1+x)^{b}
$$

to get

$$
\left(\begin{array}{c}
a+b \\
t
\end{array}\right)=\sum_{j=0}^{t}\left(\begin{array}{l}
a \\
j
\end{array}\right)\left(\begin{array}{c}
b \\
t-j
\end{array}\right) .
$$

Multiplying by $t$ ! gives the result. 
Theorem 3.2 Let sand $t$ be non-negative integers, with $s \geq t$, let $n_{0}, \ldots, n_{s}$, $m_{0}, \ldots, m_{s}$, and $\lambda_{0}, \ldots, \lambda_{t}$ be real numbers, and suppose that for $j=0, \ldots, t$, equation (2) holds. Then

$$
\sum_{i=0}^{s} P(i-x, t)\left(n_{i}-m_{i}\right)=\sum_{j=0}^{t}\left(\begin{array}{l}
t \\
j
\end{array}\right) P(-x, t-j)\left[P(s, j) \lambda_{j}-\sum_{i=j}^{s} P(i, j) m_{i}\right] .
$$

Proof. On multiplying by $j$ !, equation (2) becomes

$$
P(s, j) \lambda_{j}=\sum_{i=0}^{s} P(i, j) n_{i} .
$$

Now note that the rightmost sum in (3) can be extended to $\sum_{i=0}^{s}$, since the extra terms introduced are all zero, and so, using (4), the part in square brackets becomes

$$
\sum_{i=0}^{s} P(i, j)\left(n_{i}-m_{i}\right)
$$

Now Lemma 3.1 implies

$$
P(i-x, t)=\sum_{j=0}^{t}\left(\begin{array}{l}
t \\
j
\end{array}\right) P(i, j) P(-x, t-j) .
$$

Multiplying by $n_{i}-m_{i}$ and summing over $i$ then gives the result.

\section{Block intersection polynomials}

We call the right-hand side of (3) the block intersection polynomial for the sequences $\left[m_{0}, \ldots, m_{s}\right],\left[\lambda_{0}, \ldots, \lambda_{t}\right]$, and denote this polynomial by

$$
B(x)=B\left(x,\left[m_{0}, \ldots, m_{s}\right],\left[\lambda_{0}, \ldots, \lambda_{t}\right]\right) .
$$

Theorem 4.1 Let $D=(V, \mathcal{B})$ be a block design, let $S \subseteq V$, with $s=|S|$, and for $i=0, \ldots, s$, suppose that $m_{i}$ is a non-negative integer with $m_{i} \leq n_{i}$, where $n_{i}$ is the number of blocks intersecting $S$ in exactly $i$ points. Let $t$ be a non-negative even integer with $t \leq s$, and suppose that, for $j=0 \ldots, t$, we have $\lambda_{j}=1 /\left(\begin{array}{l}s \\ j\end{array}\right) \sum_{T \subseteq S,|T|=j} \lambda_{T}$, where $\lambda_{T}$ is the number of blocks of $D$ containing $T$.

Then the block intersection polynomial (5) is a polynomial with integer coefficients, and $B(n) \geq 0$ for every integer $n$. Moreover, if $B(n)=0$ for some integer $n$, then $m_{i}=n_{i}$ for $i \notin\{n, n+1, \ldots, n+t-1\}$. 
Proof. The hypotheses of Theorem 3.2 are satisfied, and so (3) holds; that is,

$$
\sum_{i=0}^{s} P(i-x, t)\left(n_{i}-m_{i}\right)=B(x)=B\left(x,\left[m_{0}, \ldots, m_{s}\right],\left[\lambda_{0}, \ldots, \lambda_{t}\right]\right)
$$

Since the $m_{i}$ and $n_{i}$ are integers, $B(x)$ is a polynomial over the integers. Further, note that when $t$ is even and $n$ is an integer, then $P(i-n, t)$ is a product of evenly many consecutive integers, and so is non-negative, as is $n_{i}-m_{i}$. We conclude that $B(n) \geq 0$ for every integer $n$. Moreover, since $P(i-n, t)>0$ unless $i \in\{n, n+1, \ldots, n+t-1\}$, we conclude that if $B(n)=0$, then $n_{i}=m_{i}$ for $i \notin\{n, n+1, \ldots, n+t-1\}$.

Example 1 Suppose $D$ is a 6-(14,7,4) design and $S$ is a block of $D$ (designs with these parameters are constructed in [6]). Then for this $D, S$, we take $\left[m_{0}, \ldots, m_{7}\right]=[0,0,0,0,0,0,0,1]=\left[0^{7}, 1\right], t=6$, and have

$$
\Lambda:=\left[\lambda_{0}, \ldots, \lambda_{6}\right]=[1716,858,396,165,60,18,4] .
$$

We then calculate $B(x)=B\left(x,\left[0^{7}, 1\right], \Lambda\right)=$

$$
7(x-1)\left(245 x^{5}-1222 x^{4}+3733 x^{3}-6212 x^{2}+5868 x-2160\right) .
$$

Since $B(1)=0$, we have $n_{0}=m_{0}=0$ and $n_{7}=m_{7}=1$. Thus, no block is disjoint from $S$, and $S$ is the only block meeting $S$ in all its points. This shows that each $6-(14,7,4)$ design is simple, with no two blocks disjoint. In particular, the design formed by the complements of the blocks of $D$ coincides with the design formed by the 7 -sets which are not blocks of $D$.

\section{Applying block intersection polynomials}

The calculation of block intersection polynomials and the applications described in this section are implemented in the DESIGN package (Version 1.2) [10] for GAP [4]. (The Maple system [8] was used in our initial investigations.)

We apply block intersection polynomials to determine information about the number of blocks of a block design $D$ intersecting a set of points $S$ (of size $s$ ) in a given number of points, where $S$ and possibly certain blocks of the design are already specified (this may be because of certain assumed 
properties of $D$, or in a backtrack search where the design is being built up block by block, or block-orbit by block-orbit of an assumed group of automorphisms).

The non-negative integers $m_{i}$ giving lower bounds on the numbers $n_{i}$ of blocks meeting $S$ in exactly $i$ points come from assumed or proposed properties of $D$ (we often seek to show that a proposed property leads to a contradiction), or are calculated directly using some explicitly known or proposed blocks of $D$ (such as in a backtrack search).

We need also determine, for some even $t \leq s$, the quantities $\lambda_{0}, \ldots, \lambda_{t}$ (defined in (1)) for the pair $D, S$. If $D$ is a $t$-design this is no problem. If not, then for many types of block designs, where the number of blocks, the replication number of each point and the concurrency of each pair of points are specified (see [1]), we may calculate $\left[\lambda_{0}, \lambda_{1}, \lambda_{2}\right]$ for an explicitly given set $S$ (of size at least 2).

We then calculate the block intersection polynomial (5). If this $B(x)$ does not have integer coefficients or $B(n)<0$ for some integer $n$ then the assumed or proposed properties of $D, S$ have led to a contradiction. Otherwise, if $B(n)=0$ for some integer $n$ then this tells us that for the $D, S$ with the assumed or proposed properties, if $i \notin\{n, n+1, \ldots, n+t-1\}$, we have $m_{i}=n_{i}$.

\subsection{Analyzing a block intersection polynomial}

For a block intersection polynomial $B(x)$ over the integers, it is important to be able to determine if there is an integer $n$ such that $B(n)<0$, and when there is no such $n$, then to determine the integers $n$ for which $B(n)=$ 0 . The cases of $B(x)$ being a constant polynomial or having odd degree or a negative leading coefficient are trivial. Now suppose that $B(x)$ is a non-constant polynomial of even degree over the integers and has a positive leading coefficient. We calculate an integer $b$ which is a strict upper bound on the magnitude of each zero of $B(x)$, and then need only check the value $B(n)$ for the integers $n$ with absolute value less than $b$ to determine which $n$, if any, have $B(n)=0$ or $B(n)<0$.

\subsubsection{Bounding the magnitude of a zero of a polynomial}

The bound $b$ is calculated using the following result, essentially given in the Master's thesis [9] of the second author. 
Theorem 5.1 Let

$$
f(x)=a_{d} x^{d}+a_{d-1} x^{d-1}+\cdots+a_{0}
$$

be a non-zero degree d polynomial over the reals, let

$$
g(x)=\left|a_{d}\right| x^{d}-\left|a_{d-1}\right| x^{d-1}-\cdots-\left|a_{0}\right|,
$$

and let $b$ be the least positive integer such that $g(b)>0$.

Then $b$ is greater than the magnitude of each zero of $f(x)$.

Proof. It is easy to see that if $a$ is a positive real number with $g(a)>0$, then $g^{\prime}(a) \geq 0$, where $g^{\prime}(x)$ is the derivative of $g(x)$ (in fact, $g^{\prime}(a)>0$ if $g(x)$ is non-constant). Thus, if $b$ is the least positive integer such that $g(b)>0$, we have that $g(x)$ is positive and $g^{\prime}(x)$ is non-negative on the interval $[b, \infty)$. It follows that for each complex number $z$ with $|z| \geq b$, we have

$$
|f(z)| \geq g(|z|) \geq g(b)>0 .
$$

\subsection{Bounding the multiplicity of a block in a $t$-design}

A $t$-design may have repeated blocks, and we can use a block intersection polynomial to provide an upper bound on the multiplicity of a block. Suppose $D=(V, \mathcal{B})$ is a $t-(v, k, \lambda)$ design. It is well-known that $D$ is also a $j-\left(v, k, \lambda_{j}\right)$ design for $0 \leq j \leq t$, where $\lambda_{j}=\lambda\left(\begin{array}{c}v-j \\ t-j\end{array}\right) /\left(\begin{array}{c}k-j \\ t-j\end{array}\right)$.

Now assume $t$ is even and let $p$ be the least non-negative integer for which the block intersection polynomial

$$
B\left(x,\left[0^{k}, p\right],\left[\lambda_{0}, \ldots, \lambda_{t}\right]\right)
$$

is negative for some integer value of $x$ (such a $p$ exists). Then no block $S$ of a $t$ - $(v, k, \lambda)$ design can have multiplicity $p$ or more, and so $p-1$ is an upper bound on the multiplicity of each block of a $t-(v, k, \lambda)$ design.

Example 2 Suppose $D$ is a 4 - $(23,8,6)$ design and $S$ is a block of $D$ (designs with these parameters exist $[2, \mathrm{p} .59]$ ). Then for this $D, S$, we take $t=4$, and have $\Lambda:=\left[\lambda_{0}, \ldots, \lambda_{4}\right]=[759,264,84,24,6]$. We then calculate

$$
B(x)=B\left(x,\left[0^{8}, 3\right], \Lambda\right)=36\left(21 x^{4}-106 x^{3}+291 x^{2}-366 x+140\right) .
$$


Since $B(1)=-720$, we conclude that each block of a $4-(23,8,6)$ design can have multiplicity at most 2 .

If $t$ is odd (and $k>1$ ) then we can bound the multiplicity of a block in a $t$ - $(v, k, \lambda)$ design $D$ by bounding the multiplicity of a block in a derived design of $D$, which is a $(t-1)-(v-1, k-1, \lambda)$ design. This is because a block of multiplicity $m$ in $D$ gives rise to a block of the same multiplicity in a derived design, and vice-versa. Thus, for example, a block of a 5- $(24,9,6)$ design (such designs exist [2, p.60]) can have multiplicity at most 2 .

Some constructions for non-simple $t$-designs are given in $[3,7]$, and we observe that the bound on block multiplicity described in this Section is often achieved.

\subsection{Bounding the multiplicity of a block in a resolvable $t$-design}

Now assume that $t$ is even, we have $k$ dividing $v$, and we wish to bound the multiplicity of a block in a resolvable $t-(v, k, \lambda)$ design. Now if a block $B$ is repeated $m$ times in a resolvable $t-(v, k, \lambda)$ design then $B$ is in $m$ parallel classes, and so $B$ is disjoint from at least $m(v / k-1)$ other blocks. Now let $p$ be the least non-negative integer for which the block intersection polynomial

$$
B\left(x,\left[p(v / k-1), 0^{k-1}, p\right],\left[\lambda_{0}, \ldots, \lambda_{t}\right]\right)
$$

is negative for some integer value of $x$ (such a $p$ exists). Then no block of a resolvable $t-(v, k, \lambda)$ design can have multiplicity $p$ or more, and so $p-1$ is an upper bound on the multiplicity of each block of a resolvable $t-(v, k, \lambda)$ design.

Example 3 It is unknown whether there exists a 2-(55,11,5) design [2, p.23], but applying the bound of Section 5.2 shows that in such a design each block has multiplicity at most 2 .

Suppose now $D$ is a resolvable $2-(55,11,5)$ design and $S$ is a block of $D$. Then for this $D, S$, we take $t=2$, and have $\Lambda:=\left[\lambda_{0}, \lambda_{1}, \lambda_{2}\right]=[135,27,5]$. We then calculate

$$
B(x)=B\left(x,\left[8,0^{10}, 2\right], \Lambda\right)=5\left(25 x^{2}-85 x+66\right) .
$$

Since $B(2)=-20$, we conclude that each block of a resolvable 2- $(55,11,5)$ design has multiplicity 1 . In other words, each resolvable $2-(55,11,5)$ design is simple. 


\section{References}

[1] P. J. Cameron (editor), Encyclopaedia of Design Theory, http://designtheory.org/library/encyc/

[2] C. J. Colbourn and J. H. Dinitz (editors), The CRC Handbook of Combinatorial Designs, CRC Press, Boca Raton, 1996.

[3] P. Dobcsányi, D. A. Preece and L. H. Soicher, On balanced incomplete-block designs with repeated blocks, European J. Combinatorics, to appear. Preprint available at: http://designtheory.org/library/preprints/

[4] The GAP Group, GAP - Groups, Algorithms, and Programming, Version 4.4, 2006, http://www.gap-system.org/

[5] M. Jacroux, On the block structure of proper block designs, J. Statist. Plann. Inference 9 (1984), 259-267.

[6] D. L. Kreher and S. P. Radziszowski, The existence of simple 6- $(14,7,4)$ designs, J. Combin. Theory Ser. A 43 (1986), 237-243.

[7] J. P. McSorley and L. H. Soicher, Constructing $t$-designs from $t$-wise balanced designs, European J. Combinatorics, to appear. Preprint available at: http://designtheory.org/library/preprints/

[8] Maple, Version 9.5, 2004, http://www.maplesoft.com/

[9] L. Soicher, The Computation of Galois Groups, M.Comp.Sci. thesis, Concordia University, Montreal, 1981.

[10] L. H. Soicher, The DESIGN package for GAP, Version 1.2, 2006, http://designtheory.org/software/gap_design/ 